\title{
BIOLOGICAL ACTIVITY OF NITROGUAIACOL ETHER DERIVATIVES OF STREPTOMYCIN
}

\author{
José P. Abad, Gloria LeÓN ${ }^{\dagger}$ and Ricardo Amils* \\ Centro de Biología Molecular (CSIC-UAM) Universidad Autónoma, \\ Canto Blanco, 28049 Madrid, Spain \\ Instituto de Bioquímica, Universidad Austral de Chile, \\ Casilla 567, Valdivia, Chile
}

(Received for publication May 20, 1986)

\begin{abstract}
The correlation between the "in vivo" and "in vitro" inhibitory properties of six nitroguaiacol ether derivatives of streptomycin is studied. The differential activity of the derivatives is more closely related to differences in efficiency in the transport of the drugs than to their capacity to inhibit poly $(U)$ directed polyphenylalanine synthesis. The results obtained are discussed in relation to the characteristics of the different derivatives.
\end{abstract}

Streptomycin $(\mathrm{Sm})$, one of the first aminoglycoside antibiotics used in pharmacology, has been very important in the treatment of tuberculosis. After several years of clinical use in a wide range of infectious diseases, streptomycin was replaced by other drugs because: i) its selective toxicity on the VIII craneal nerve ${ }^{1 \sim 4)}$ and ii) the selection of resistant strains some of them harboring inactivation systems $^{5,8)}$. Several attempts to obtain derivatives of this antibiotic ${ }^{711)}$ with higher activity or lower sensitivity to inactivation enzymes were made, but the lack of a systematic survey of their biological properties made it difficult to design new drugs with better pharmacological characteristics.

In this paper we report the biological properties of different nitrophenylether derivatives of streptomycin (Fig. 1), which open up the possibility of designing newer derivatives of this important protein synthesis inhibitor.

Fig. 1. Comparative structures of the reduced carbohydrazone derivatives of streptomycin.

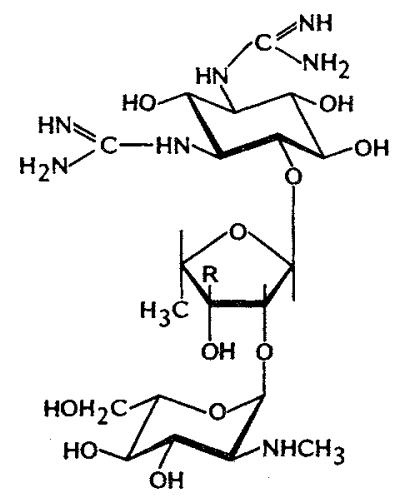

$$
\text { Sm }
$$

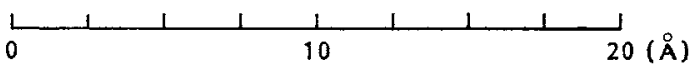




\section{Materials and Methods}

\section{Streptomycin Derivatives}

The derivatives used in the present work were synthesized according to ABAD et al. ${ }^{12}$.

Bacterial Strains and Growth Conditions

Escherichia coli MRE 600 and Bacillus subtilis ATCC 6633 were grown at $37^{\circ} \mathrm{C}$ in a medium containing yeast extract $5 \mathrm{~g} /$ liter Bacto-tryptone $10 \mathrm{~g} /$ liter, $\mathrm{NaCl} 5 \mathrm{~g} /$ liter and D-glucose $0.2 \%$. Petri dishes were prepared with the same medium without glucose and with $1 \%$ soft agar.

Ribosomes and Soluble Fraction

The method described by AmiLs et al. was followed ${ }^{13)}$.

Inhibitory Assay "In Vivo"

Three methods were used to test inhibition of growth of E. coli and B. subtilis: i) In Petri dishes, $10 \mathrm{~cm}$ in diameter, $5 \mathrm{ml}$ of rich media with $1 \%$ soft agar were mixed with $5 \mathrm{ml}$ of bacteria in the exponential phase of growth at an optical density of 0.005 at $660 \mathrm{~nm}$. $3 \mathrm{MM}$ Whatman paper discs, $4 \mathrm{~mm}$ in diameter, containing different concentrations of the antibiotic to be tested, were placed on the agar. After incubation at $37^{\circ} \mathrm{C}$ overnight the halos of inhibition were measured.

ii) A more precise measurement of the inhibitory effect was performed by following the growth of the cells in $25-\mathrm{ml}$ Erlenmeyer flasks, starting with suspensions of cells at an optical density of 0.1 at $660 \mathrm{~nm}$. Different concentrations of the inhibitors were added and the growth followed by optical density measurements. The inhibitory effect was calculated by comparison with a control with no antibiotic.

iii) For a better comparative survey of the different derivatives multi-inhibitory experiments were performed in microcultivation plastic racks (Flow Lab. Inc.) with 96 wells. The microcultures were incubated at $37^{\circ} \mathrm{C}$ in $200 \mu 1$ volume. The growth was followed by automatic determination of the optical density at $450 \mathrm{~nm}$ in a Titertek Multiskan (Flow Lab. Inc.) in which all 96 samples can be measured in less than 1 minute.

Competition Experiments with $\left[{ }^{3} \mathrm{H}\right]$ Dihydrostreptomycin $\left(\left[{ }^{3} \mathrm{H}\right] \mathrm{DHSm}\right)$

The competition experiments were done following the method of CHANG and FLAKs ${ }^{14)}$.

Poly(U) Directed Poly(Phe) Synthesis

"In vitro" protein synthesis was done in accordance to AMILs et al. ${ }^{13)}$.

Determination of the Transport of Radioactive Derivatives

Exponentially growing $E$. coli cells diluted to an optical density of 0.1 at $660 \mathrm{~nm}$ were incubated with radioactive derivatives of streptomycin at a concentration of $2 \mu \mathrm{g} / \mathrm{ml}$ (specific radioactivity 500 $\mathrm{cpm} / \mathrm{pmol}$ ). The cells were grown for 100 minutes at $37^{\circ} \mathrm{C}$, harvested by centrifugation at low speed and washed with an ice-cold solution of $0.1 \mathrm{M} \mathrm{LiCl}$ containing $20 \mu \mathrm{g} / \mathrm{ml}$ of unlabeled streptomycin. After three washing cycles the cells were resuspended in buffer NB (10 mM Tris- $\mathrm{HCl}, \mathrm{pH} 7.4 ; 10 \mathrm{~mm}$ $\mathrm{MgAc}_{2}, 60 \mathrm{mM} \mathrm{NH}_{4} \mathrm{Cl}$ and $6 \mathrm{~mm} \beta$-mercaptoethanol), and the optical density and the radioactivity incorporated were measured. Aliquots of control cells grown in the absence of antibiotic were used as blanks. Appropriate control showed that the addition of radioactive derivatives during the harvesting and washing of the cells does not produce any significant incorporation of radioactivity.

\section{Results and Discussion}

Three carbohydrazone derivatives of streptomycin with different length spacers between the nitroguaiacol group and the carbonyl of streptomycin with their corresponding reduction products were synthesized as described by ABAD et al. ${ }^{12}$. These derivatives inhibit the growth of Gram-negative eubacteria (Escherichia coli) and Gram-positive eubacteria (Bacillus subtilis) with differing efficiencies. Fig. 2 depicts the inhibition curves exhibited by the different derivatives in both types of bacteria: 
Fig. 2. Growth inhibition produced by the streptomycin derivatives in Escherichia coli (A) and Bacillus subtilis (B).

- Sm, O DHSm, $\mathrm{G}_{1} \mathrm{Sm}, \square \mathrm{DHG}_{1} \mathrm{Sm}, \mathbf{\mathrm { A }} \mathrm{G}_{4} \mathrm{Sm}, \triangle \mathrm{DHG}_{4} \mathrm{Sm}, \nabla \mathrm{G}_{8} \mathrm{Sm}, \nabla \mathrm{DHG}_{8} \mathrm{Sm}$.

(A)

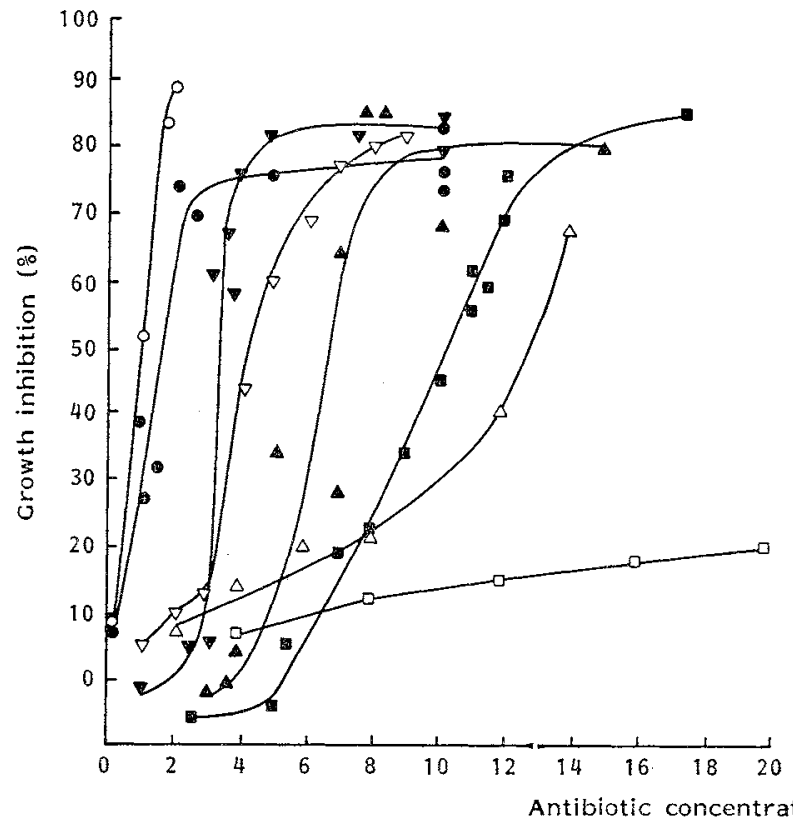

(B)

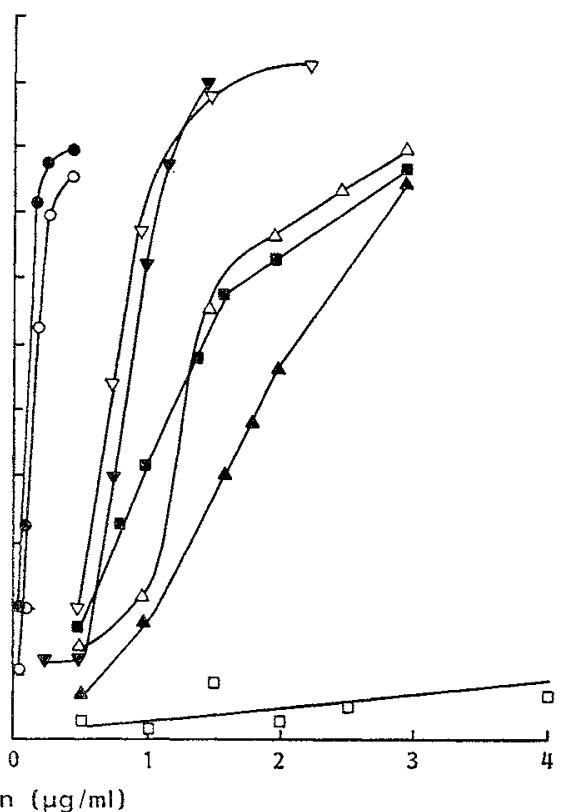

E. coli $\quad \mathrm{DHG}_{1} \mathrm{Sm} \ll \mathrm{DHG}_{4} \mathrm{Sm}<\mathrm{G}_{1} \mathrm{Sm}<\mathrm{G}_{4} \mathrm{Sm}<\mathrm{DHG}_{8} \mathrm{Sm} \leq \mathrm{G}_{8} \mathrm{Sm}<\mathrm{DHSm} \leq \mathrm{Sm}$

B. Subtilis $\quad \mathrm{DHG}_{1} \mathrm{Sm} \ll \mathrm{G}_{4} \mathrm{Sm}<\mathrm{DHG}_{4} \mathrm{Sm}=\mathrm{G}_{1} \mathrm{Sm}<\mathrm{DHG}_{8} \mathrm{Sm}=\mathrm{G}_{8} \mathrm{Sm}<\mathrm{DHSm}=\mathrm{Sm}$

These results show that the activity of the derivatives is proportional to the length of the methylene chain located between the antibiotic and the bulky aromatic group. This finding may explain why most of the derivatives reported in the literature are inactive. Almost all of them were obtained by modifying the carbonyl group of streptomycin with bulky structures attached by short spacers ${ }^{87}$. Although the general inhibition pattern is maintained for both cell types for the nitroguaiacol derivatives, some differences can be detected. As expected the Gram-positive B. subtilis is much more sensitive than the Gram-negative $E$. coli. The inhibitory activity of $\mathrm{DHG}_{4} \mathrm{Sm}$ and $\mathrm{G}_{1} \mathrm{Sm}$, which is lower than $\mathrm{G}_{4} \mathrm{Sm}$ in $E$. coli, is higher in $B$. subtilis. All the derivatives assayed are less active than streptomycin and dihydrostreptomycin.

The difference in activity displayed by the derivatives could be explained by any of the following hypothesis:

i) Affinity of the derivatives for their target sites, ii) efficiency of the streptomycin transport system or iii) secondary effects other than protein synthesis inhibition.

As mentioned before streptomycin is considered a specific inhibitor of protein synthesis ${ }^{15)}$ requiring a specific binding site to produce its inhibitory effects ${ }^{16,17)}$. Streptomycin resistant mutants have been characterized as having modified ribosomal proteins or $\mathrm{RNA}^{18 \sim 21)}$. In order to test the ability of these derivatives to bind to sensitive ribosomes, competition experiments with $\left[{ }^{3} \mathrm{H}\right] \mathrm{dihy}-$ drostreptomycin were performed. Fig. 3 shows that all the derivatives are able to compete with radioactive dihydrostreptomycin for the same binding site, although their affinities for this site are dif- 
Fig. 3. Competition between $\left[{ }^{3} \mathrm{H}\right] \mathrm{DHSm}$ and the streptomycin derivatives for their binding site in Escherichia coli ribosome.

A $0.3 \mu \mathrm{M}$ solution of $70 \mathrm{~S}$ ribosomes from $E$. coli was incubated at $37^{\circ} \mathrm{C}$ for 45 minutes under appropriate ionic conditions with $1.1 \mu \mathrm{M}\left[{ }^{3} \mathrm{H}\right] \mathrm{DHSm}$ and different concentrations of the derivatives. The binding of the tritiated DHSm was estimated by filtration as described in Materials and Methods.

(A) $\bigcirc \mathrm{Sm}, \wedge \mathrm{G}_{1} \mathrm{Sm}, \mathrm{G}_{4} \mathrm{Sm}, \mathbf{\mathrm { m }} \mathrm{G}_{8} \mathrm{Sm}$.

(B) $\bigcirc \mathrm{DHSm}, \wedge \mathrm{DHG}_{1} \mathrm{Sm}, \bullet \mathrm{DHG}_{4} \mathrm{Sm}, \mathbf{D H G} \mathrm{Sm}$.

(A)

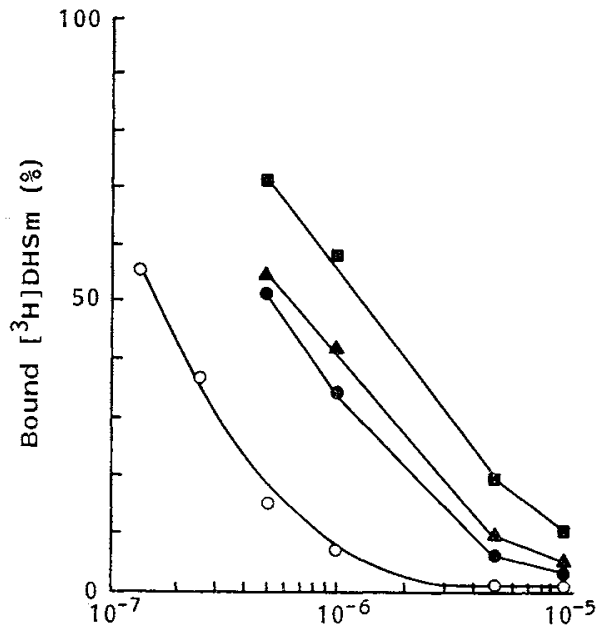

(B)

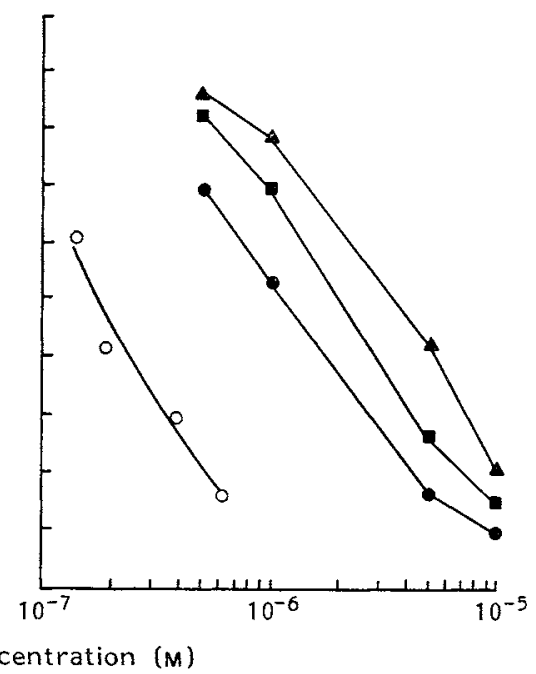

Fig. 4. Inhibition of the poly(Phe) synthesis directed by poly(U) produced by the different streptomycin derivatives in the Escherichia coli system.

A solution of $0.1 \mu \mathrm{M}$ of $E$. coli ribosomes was incubated in the conditions described in Materials and Methods with different concentrations of derivatives of streptomycin. The percentage of inhibition was calculated by comparing the amount of radioactive poly(Phe) synthesized in the absence of antibiotic. Panel A non-reduced and panel $\mathbf{B}$ reduced derivatives.

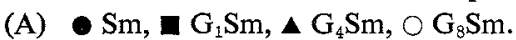

(B) $\bullet \mathrm{Sm}, \boldsymbol{\sim} \mathrm{DHG}_{1} \mathrm{Sm}, \wedge \mathrm{DHG}_{4} \mathrm{Sm}, \bigcirc \mathrm{DHG}_{8} \mathrm{Sm}$.

(A)

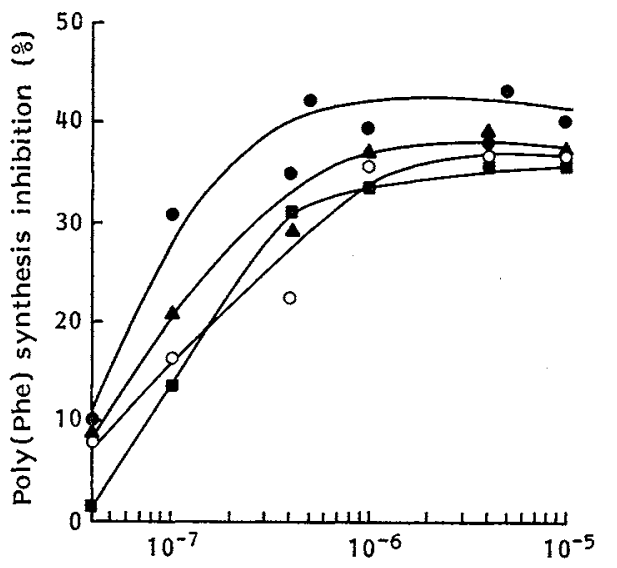

(B)

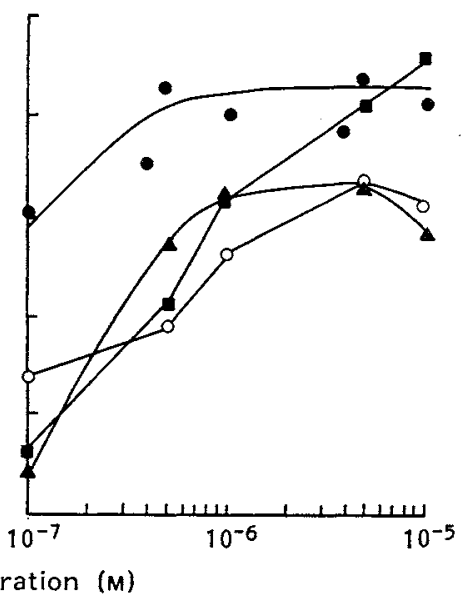


ferent. The reduced derivatives compete with lower efficiency than the non-reduced ones, and among those the derivatives with shorter spacers $\left(\mathrm{G}_{4} \mathrm{Sm}\right.$ and $\left.\mathrm{G}_{1} \mathrm{Sm}\right)$ are more efficient competitors. The affinity pattern for the ribosome $\mathrm{DHG}_{1} \mathrm{Sm}<$ $\mathrm{DHG}_{8} \mathrm{Sm}<\mathrm{G}_{8} \mathrm{Sm}<\mathrm{DHG}_{4} \mathrm{Sm}<\mathrm{G}_{1} \mathrm{Sm}<\mathrm{G}_{4} \mathrm{Sm}<$ $\mathrm{DHSm}=\mathrm{Sm}$ is quite different from the inhibition produced by the derivatives in "in vitro" experiments.

The effect of these derivatives on the inhibition of poly(Phe) synthesis directed by poly(U), shown in Fig. 4, basically agrees with the com-
Table 1. Transport efficiency of the reduced derivatives of streptomycin in the Escherichia coli system.

Early $\log$ phase $E$. coli cells were grown in the presence of $2 \mu \mathrm{g} / \mathrm{ml}$ of the different radioactive reduced derivatives of streptomycin, in the conditions described in Materials and Methods. After 100 minutes of incubation at $37^{\circ} \mathrm{C}$ the amount of radioactivity incorporated into the cells was determined.

\begin{tabular}{cc}
\hline & $\begin{array}{c}\text { pmol } / \text { unit of } \\
\mathrm{A}_{860} \times \mathrm{ml} \text { of culture }\end{array}$ \\
\hline$\left[{ }^{3} \mathrm{H}\right] \mathrm{DHG}_{1} \mathrm{Sm}$ & 3.0 \\
{$\left[{ }^{3} \mathrm{H}\right] \mathrm{DHG}_{4} \mathrm{Sm}$} & 9.2 \\
{$\left[{ }^{3} \mathrm{H}\right] \mathrm{DHG}_{8} \mathrm{Sm}$} & 20.4 \\
\hline
\end{tabular}
petition experiments discussed above, indicating that the differential "in vitro" inhibition on protein synthesis is a reflection of the affinity of the derivatives for their target site.

It is obvious from these results that the inhibition levels in "in vivo" experiments cannot be attributed to differential effects on protein synthesis, because there is no strict correlation between the "in vivo" and "in vitro" assays.

To test the second hypothesis, radioactive derivatives were used to measure the relative rate of accumulation in $E$. coli, under conditions were no damage of the membrane could be detected. Under these conditions the accumulation of radioactive derivatives in the cell gives a rough estimate of the transport system's efficiency. The results in Table 1 show that accumulation of the three derivatives is different and the sequence and order of magnitude agrees with the inhibitory effects observed in the "in vivo" experiments.

Although we cannot rule out the possibility that these streptomycin derivatives can produce secondary or nonspecific effects that are not related to transport or protein synthesis, we do not believe that they play an important role in the differential effects observed in these experiments.

The properties that the differences in the length of the spacers confer on the derivatives and which should be considered when explaining their differential inhibitory effects are at least: i) The increase in the hydrophobicity of the molecule due to an increase in the number of methylene groups and ii) the increase in the distance between the antibiotic and the nitroguaiacol group affecting the steric hindrance of the bulky modification of the antibiotic.

In the case of transport, both variables, hydrophobicity and distance increase in the same direction as the results observed in the "in vivo" experiments. Although the hydrophobicity of each derivative and the corresponding reduced products should be similar, their activities are noticeably different, suggesting that the differences resulting from the transport of the drugs might be more related to steric hindrance than to hydrophobicity. The free rotation around the reduced $\mathrm{C}-\mathrm{N}$ bond could be responsible for the larger steric hindrance of the bulky group when compared to the homologous non-reduced derivatives. This effect should weaken as the length of the spacer increases. When model systems are constructed, similar steric hindrances are obtained for $\mathrm{DHG}_{8} \mathrm{Sm}$ and $\mathrm{G}_{8} \mathrm{Sm}$ derivatives, due to the different possible conformations of the flexible methylene chain. When the length of the spacer is shorter, the effect of reducing the double bond should be greater. This is precisely what is observed in the "in vivo" experiments. In the case of the shorter spacer derivatives $\mathrm{G}_{1} \mathrm{Sm}$ and $\mathrm{DHG}_{1} \mathrm{Sm}$, this effect is very important producing a dramatic reduction of the activity of the non-reduced derivative. 
Concerning the interaction of the streptomycin with its target site similar considerations can be made although it seems that hydrophobicity is as important as the steric hindrance effects already described. In this case the difference between the derivatives is not as dramatic as in the transport system. This may be due to the characteristics of the interaction with the ribosome. If we assume that the "in vitro" affinities measured reffect the "in vivo" affinities of the derivatives for the ribosomes, our results suggest that the limiting rate for the effective action of these derivatives is the transport step in BRYAN's model for the mode of strepotmycin action ${ }^{22)}$. In any case we feel that although these derivatives could be used to study the different models of streptomycin effect, many variables must be measured before any conclusion can be drawn.

Using these derivatives we have mapped the binding site of streptomycin by photo-affinity labeling sensitive ribosomes with these nitroguaiacol ether derivatives. The results locate the interaction site on the interface between the subunits in a position where bulky groups do not interfere with the access of the antibiotic to the binding site $^{23)}$.

Results describing streptomycin derivatives with potential pharmacological applications cannot be easily interpreted. We believe that a more rational and systematic exploration of the effect of different modifications on the streptomycin may lead to new derivatives with improved properties. In our laboratory we are currently synthesizing streptomycin derivatives with different modifications (effectors, spacers, linkage reactions, etc.) in order to test their effect on the different steps responsible for the inhibitory effect of the antibiotic; transport, inactivation, interaction with the target, side effects, etc. We hope that this approach will clarify our hypothesis regarding these derivatives and eventually help in the design of new semi-synthetic derivatives of streptomycin with useful pharmacological applications.

\section{Acknowledgments}

This work has been supported by grants from the CAICYT and by an institutional grant from the Fondo de Investigaciones Sanitarias (FIS). We thank Antibióticos S.A. for their collaboration, and Dr. J. P. G. BALIESTA and D. VÁzQuez for many valuable discussions. One of us, J. P. A., was a predoctoral fellow from the Fondo de Investigaciones Sanitarias.

\section{References}

1) BERG, K.: The toxic effect of streptomycin on the vestibular and cochlear apparatus. Acta OtoLaryngol. (Suppl) 97: 1 47, 1951

2) MoRIYAMA, S.: Studies on streptomycin deafness in its early stage. J.P.N. Otolaryngol. 62: 107 108, 1959

3) Weinstein, L.: Streptomycin. In The Pharmacological Basis of Therapeutics. Eds., L. S. Goodman \& A. Grlman, Chapter 58, The MacMillan Co., London, 1971

4) KoEda, T.; K. UmeMURA \& M. YoKota: Toxicology and pharmacology of aminoglycoside antibiotics. In Aminoglycoside Antibiotics. Handbook of Experimental Pharmacology. Vol. 62. Eds., H. UMEZAwA \& I. R. HOOPER, pp. 293 356, Springer-Verlag, Berlin, 1982

5) Benveniste, R. \& J. Davies: Mechanisms of antibiotic resistance in bacteria. Annu. Rev. Biochem. 42: $471 \sim 506,1973$

6) UmeZawa, S. \& S. Kondo: Mechanisms of resistance of aminoglycoside antibiotics. In Aminoglycoside Antibiotics. Handbook of Experimental Pharmacology, Vol. 62. Eds., H. UMEZAWA \& I. R. Hooper, pp. 267 292, Springer-Verlag, Berlin, 1982

7) Umezawa, S. \& T. TSuchiYa: Total synthesis and chemical modification of the aminoglycoside antibiotics. In Aminoglycoside Antibiotics. Handbook of Experimental Pharmacology. Vol. 62. Eds., $\mathrm{H}$. UMEZAWA \& I. R. HOOPER, pp. 37 97, Springer-Verlag, Berlin, 1982 
8) Golubev, V.N.: Salts and other synthetic derivatives of streptomycin and dihydrostreptomycin. Antibiotics (USSR) 12:1119 1129, 1967

9) Heding, H. \& O. LÜtzen: Streptomycin: Correlation between in vivo and in vitro activity of ten representative derivatives. J. Antibiotics 25: 287 291, 1972

10) Treffers, H. P.; N. O. Belser \& D. C. Alexander: Genetic studies on resistance to streptomycin and to substituted streptomycin $\mathrm{N}^{\prime}$-(3-hydroxypyropyl) streptomycylamine. Antibiotic Ann. 1953/54: 595 603,1953

11) Treffers, H. P. \& D. C. Alexander: The effect of chemical substitution on the activity and resistance patterns of streptomycin derivatives. Antibiotic Ann. 1955/56: 802 809, 1955

12) ABAD, J. P.; G. LEON \& R. AmIls: Synthesis of active nitroguaiacol ether derivatives of streptomycin. J. Med. Chem., to submitted

13) Amils, R.; E. Mathews \& C. Cantor: Reconstitution of 50 S ribosomal subunits from E. coli. In Methods in Enzymology. Vol. 59. Eds., S. P. Colowrck \& N. O. KaPlan, pp. 449 461, Academic Press, New York, 1979

14) Chang, F. N. \& J.G. Flaks: Binding of dihydrostreptomycin to Escherichia coli ribosomes: Kinetics of the reaction. Antimicrob. Agents Chemother. 2: 308 319, 1972

15) ERdös, T. \& A. UlLMANN: Effect of streptomycin on the incorporation of amino-acids labelled with carbon-14 into ribonucleic acid and protein in a cell-free system of a mycobacterium. Nature 183: 618 619,1959

16) Spotts, C. R. \& R. Y. Stanier: Mechanism of streptomycin action on bacteria: a unitary hypothesis. Nature 192: 633 637, 1961

17) Cox, E. C.; J.R. White \& J. G. Flaks: Streptomycin action and the ribosome. Proc. Natl. Acad. Sci. U.S.A. $51: 703 \sim 704,1964$

18) DAvites, J. E.: Studies on the ribosomes of streptomycin sensitive and resistant strains of Escherichia coli. Proc. Natl. Acad. Sci. U.S.A. 51 : 659 664, 1964

19) Ozaki, M.; S. Mizushima \& M. Nomura: Identification and functional characterization of the protein controlled by the streptomycin-resistant locus in E. coli. Nature 222: 333 339, 1969

20) DabBs, E. R. \& H. G. Wirtmann: A strain of Escherichia coli which gives rise to mutations in a large number of ribosomal proteins.' Mol. Gen. Genet. 149: 303 309, 1976

21) Montandon, P.; P. Nicolas, P. P. Schurmann \& E. Stutz: Streptomycin-resistance of Euglena gracilis chloroplasts: Identification of a point mutation in the 16S rRNA gene in an invariant position. Nucleic Acids Res. 13: 4299 4310, 1985

22) BRYAN, L. E. \& S. KWAN: Roles of ribosomal binding, membrane potential, and electron transport in bacterial uptake of streptomycin and gentamicin. Antimicrob. Agent Chemother. 23: 835 845, 1983

23) ABAD, J. P.: Synthesis and biological activity of photoreactive derivatives of streptomycin. Its use in the study of the binding site of the antibiotic. Ph. D. Thesis, Univ. Autónoma de Madrid, 1985 